\title{
Critical Thinking Development in College English Teaching
}

\author{
Cong $\mathrm{Li}^{1, \mathrm{a}}, \mathrm{Li} \mathrm{Jian}^{2, \mathrm{~b}^{*}}$ \\ ${ }^{1}$ Department of Foreign Languages, The Dalian Jiaotong University, Dalian, Liaoning, China \\ ${ }^{2}$ Department of Art, The Dalian Jiaotong University, Dalian, Liaoning, China \\ a184532585@163.com \\ b*258792354@163.com
}

\begin{abstract}
Critical thinking ability is very significant for Higher Education. Since the cultivation of critical thinking can be traced throughout the history of western and Chinese education, it should still remain one of the fundamental goals of College education. Due to the dramatic changes brought by globalization and Convid- 19 pandemics, cross-cultural interaction and mutual understanding seem more meaningful for the whole world. As an important humanistic course, College English is a required course for all non-English major students and exerts great impacts on cultivating college students critical thinking competence. There are many suggestions on how the English teachers integrate critical thinking into teaching practice.
\end{abstract}

Keywords: College English, critical thinking, teaching practice, cognitive goals

\section{大学英语课程中的思辨教学}

\author{
从莉 ${ }^{1, a}$, 李健 ${ }^{2, b *}$
}

${ }^{1}$ 大连交通大学外国语学院, 大连, 辽宁, 中国

大连交通大学艺术学院, 大连, 辽宁, 中国

a amyxianroy@aliyun.com

b*lianjianysx@163.com

\section{摘要}

在高等教育中思辨能力的培养至关重要，在中西方的教育历史上对思辨能力的培养始终贯穿其中。尤 其当今世界处于全球化，反全球化以及新冠疫情给世界带来的巨大变革中，跨文化交际、跨文化互信 和理解变得尤为重要。思辨能力培养与跨文化意识培养有密切关联。大学英语课程作为大学重要的人 文通识课程, 开课面广, 在高等院校培养体系中, 尤其是跨文化沟通能力和思辨能力培养方面发挥着 重要作用。因此十分有必要探寻在大学英语课程设计中如何解构思辨能力和如何在大学英语课程中融 入思辨能力培养的教学环节, 使课堂教学能够有效地实现教学目标。

关键词: 大学英语, 思辨能力, 教学实践, 认知目标

\section{1. 思辨能力概述和研究的缘起}

思辨能力（critical thinking）是源自西方的概 念。对思辨能力的定义和解释在漫长的教育发展史上不 同时期的不同学者都做过角度不同的阐述。从广义的角 度来定义, 思辨能力就是“在标准之上有辨别力的判断” (Foundation，2011），但这种解释过于笼统, 无法具 体分解出思辨能力的维度和构成, 很难测量并把培养思 辨能力付诸于教育实践, 因此 1990 年, 美国哲学协会 发布了《德尔菲报告》（Delphi Report）从两个维度即
认知技能和情感意向对思辨能力进行解构。认知技能是 “一种基于诠释、分析、评价、推断、解释和自我调节 等技能的判断, 也是一种基于证据、概念、方法、标准 和语境的判断”, 这一解释强调从认知的角度, 思辨能 力需要一些技巧和策略来达到进行判断的目的; 情感意 向则是 “是一种态度或性情, 使人能够意识到何时需要 运用某种技能以及愿意去运用这些技能”。(林晓\&何莲 珍, 2017) 这种情感意向维度强调思辨能力应用的情境, 也就是在何时何地何境来进行思辨。两个维度相加把思 辨能力放置在具体的情境中, 并从目的、策略和技能等 方面构建了思辨能力的框架, 为在现实的教学或课程中 培养思辨能力提供了路径。 
事实上, 思辨能力的培养一直是中外教育中不可或 缺的重要组成部分, 思辨能力的教育形式在教育发展的 历史中不断的演化和发展。尤其是在高等教育出现之后, 思辨能力一直是高等教育中的核心目标及要素。当今时 代世界所处的格局是以往从未有过的, 全球化和后信息 化时代使知识的产生、传递和创新方式都发生了翻天覆 地的变化, 以知识为驱动的新经济时代使世界各国都在 不得不面对全球化带来的政治、经济等新挑战的同时, 也不得不在合作、共荣、摩擦和矛盾中重新认识和妥善 处理自身与他国, 自身与世界的关系。而未来各国竞争 的关键必然是人才之争, 因此各国高等教育对人才培养 的目标不再是只针对本国的情况和培养只适应本国需 求的人才, 而是要培养能够在纷繁复杂的国际环境中, 有明晰判断, 能够冲破狭监, 能够走向团结合作的新一 代人才。而能够在复杂的情境中做出合理的分析、判断 的能力即思辨能力。尤其是在跨文化领域, 思辨能力的 培养, 对于所培养出来的人才是否在全球沟通、合作中 能够理性的做出判断, 真正具有积极的跨文化意识更具 有重要意义。因此高等院校应进一步探寻如何将思辨能 力培养融合到日常教学中, 从教学实践的角度找到有效 的路径。作为培养学生跨文化意识的重要通识课程, 大 学英语课程在整个人才培养体系中应发挥积极的作用。 在大学英语课程中明确思辨能力培养目标不仅是课程 性质和课程目标的必然要求, 也是培养未来具有国际视 野, 能够引领国家复兴, 实现人类命运供体终极目标的 必然要求。

\section{2. 思辨能力培养在教育史上的发展和演变}

\section{1. 西方教育中的思辨能力培养}

思辨能力培养在西方教育史上源远流长, 最早可从 古希腊教育的萌芽期开始追溯。从苏格拉底时代开始, 教学领域中就有思辨教学的影子。苏格拉底认为, 知识 和美德是能通过受教育而得到的, 而他通过一种 “产婆 术” 的方式来进行教学。产婆术主要是通过辩论和追问 等方法揭示矛盾, 解决矛盾, 从而获得知识的一种方法。 它基本的步骤和方法是通过不断追问直至让提问者发 现自身逻辑和想法中的矛盾和不足; 然后通过举例、提 示等方法引导学生进行思考, 发现不足的原因并试图找 到解决问题的答案。这是类似于 “助产” 的教学方法; 在找到答案后教师再引导学生从具体事物出发, 归纳总 结出一般规律, 这一过程已经上升到知识建构层面, 采 用的是 “归纳” 方法; 最后在归纳之上, 再引导学生对 一般规律产生清楚的认识并给出明确阐述。这是 “定义” 的方法, 通过下定义使思维更加清晰明确。可以看出, 苏格拉底的教学是从不断地追问开始, 通过判断、纠正 找出矛盾并解决, 最终得到问题的答案, 从而获取新知 识、产生新的知识架构。此时提问一反驳一分析一判断等过 程就是思辨能力培养的过程。思辨法在此时是知识产生 的起点, 也是达到知识获得的一种方法。
在中世纪的欧洲, 大学初见端倪。萨莱诺大学、博 洛尼亚大学和巴黎大学等一批典型的大学建立起来, 教 师的管理主要由一些行会进行, 教学以单一学科独立进 行。而当时普遍的教学组织模式主要是读课、辩论、游 历和师徒制。其中读课就是教师选择本学科的经典读物, 全书通读给学生, 学生进行记录。其中, 读课是知识的 传递和输入阶段, 而重要的学习建构构成过程主要是辩 论和游历。辩论是教师组织学生就在读课过程中所学内 容进行思考、提问和辩论, 通过一种思辨的过程促进学 生对所学内容进行理解和思考, 同时通过师生之间、学 生之间的争论等促进反思、分析和判断以便产生新知识, 建构新的知识结构。之后通过游历的方式扩展知识来源, 进一步发展学生的现实世界的感知, 拓展知识体系。辩 论课是当时大学里重要的学习和学术活动, 分为不同层 次, 目的不同并在不同的周期进行, 全体学生均要参加。

文艺复兴时期人文主义开始进入到教育领域, 教育 的重心开始关注人的全身心发展，此时的教育更加注重 教育中对人思维的启发和对思考能力的培养。欧洲此时 从禁锢的神学宗教黑暗期走向人文主义和自然主义。17 世纪夸美纽斯在他的著作《大教学论》和《教学法解析》 论述了教学思想, 是对这一时期较好的总结, 并且奠定 了现代教学的基本框架。在这些著作中, 他提出学术能 力的训练应该采用公开讨论的方法, 教授对一个议题的 讲解, 应该把关于这一议题最好的作品发给学生。由教 师先教授与议题相关的内容, 然后学生准备一个问题和 观点, 其他学生可以参与讨论, 证明观点正确与否。教 师作为辩论主持人给与总结, 并以此提出学位授予的答 辩制度。

19 世纪洪堡大学的成立标志着现代大学的成立。培 养学生创新能力和创新性思维一直是现代大学的重要 目标。而创新能力的重要组成部分就是思辨能力。德国 洪堡大学开始, 就采用了讨论课的教学和课程模式 （seminar）。讨论课最早由是 18 世纪的哥廷根大学 开设的, 主要以学生就所学的主题和内容收集资料, 从 资料中寻找证据和知识, 形成个人的观点, 解决对相关 议题的困惑, 清晰阐述观点, 并通过师生间的讨论最终 获得明确的认识。而这种讨论课的教学形式一直保留到 今天, 在现代西方大学中讨论课仍然是主要的课程组织 形式之一。

2011 年美国大学协会调查了 433 家高等教育机构, 结果显示 $95 \%$ 的首席学术长把思辨能力列为大学生最应 该掌握的学术技能。（马晓花\&龚晓斌，2019）

由此可见思辨能力培养是贯穿西方教育始终, 它是 重要的教学形式, 也是教育的重要目标之一。

\section{2. 中国传统教育中的思辨能力培养}

中国的教育思想中一直重视思辨能力对知识建构 的影响。在论语中就有“学而不思则目, 思而不学则殆。” 的论述, 强调思考与知识习得的关系。理学之集大成者 朱喜也在能够阐述其教育思想的《白鹿洞书院学规》中 提出 “审问之。慎思之。明辨之。笃行之。”王阳明认 
为: “夫学贵得之心, 求之于心而非也, 虽其言出自于 孔子, 不敢以为是也。” 都强调学习需要有批判性思维, 应该培养学习者思辨的能力。到了明末, 清末对八股文 的废止, 改用策论代替, 也是教育改革中重视思辨能力 培养的重要表现。

现代高等教育中也注重对学生思维和创新能力的 培养。《高等教育法》中明确指出要培养 “创新精神” 的高级人才。

\section{3. 大学英语教学中进行思辨教学的必然性及 现状}

大学英语课程是高等院校重要的人文通识课程, 兼具人文性和工具性双重性质。在 2017 年教育部颁 布的最新版《大学英语教学指南》中, 虽然其中没有 明确提出把培养思辨能力作为大学英语课程教学目 标, 但大学英语课程一个重要目标就是要培养学生 “跨文化意识”, 要 “增进对不同文化的理解、对中 外文化异同的意识, 培养跨文化交际能力。” 而跨文 化意识的重要组成部分就是思辨能力, 只有培养好学 生的思辨能力才能够在不同的情境下采用正确的策 略, 对所遇到的问题进行分析和判断, 对跨文化交际 的过程中遇到的文化现象、矛盾、冲突、文化异同等 进行分析、判断并采取相应的策略保持跨文化沟通顺 畅。思辨能力是人文通识课程的基本教育目标, 应该 涵盖在大学英语课程目标之内。尤其 2020 年全球爆 发新冠疫情, 各国对疫情的处理方式, 人们对疫情所 带来问题的判断以及疫情对人们价值观、世界观的冲 击都引人深思。思辨能力的缺位让一些国家陷入混乱, 人们对疫情不科学的认识以及不同文化、政治体制之 间的异同被政治化甚至共同抗疫的有益举措也被污 名化, 这些现象引起了世界范围内广泛的担忧。面对 世界范围内的文化冲突升级, 如何认识世界格局, 如 何看待全球化和人们共同命运, 将是未来相当长一段 时间内各国高等教育应该关注的重要问题。而在这种 局面下, 中国的高等教育, 更应该关注如何培养优秀 的人才。使其具有清晰的价值观和世界观, 有能力透 过纷繁复杂的表象看到事物的本质和发展规律, 使其 能够在政治、文化冲突中发展自身的价值判断, 有能 力辨别、分析并得出正确的结论, 真正具有积极意义 的跨文化意识, 增强对外跨文化沟通能力。要实现以 上的教学目标, 大学英语课程具有不可取代的作用。 之所以大学英语被设置成为面向除英语专业以外各 专业学生必修课程, 就是要在各类人才培养过程中, 不仅使学生具备一定的语言技能, 而且还要具有国际 化的视野和跨文化沟通能力, 使新一代的建设人才能 够让中国更好的应对越来越复杂的国际局势。

当前针对大学英语课程面临的使命, 已经有一些 教师和学者认识到思辨教学的重要性, 并探讨如何在
教学中融入思辨能力培养的内容。如从英语写作方面 探讨培养学生思辨能力的 (金晓宏, 王婷, 周一书); 也有学者从听力, 阅读等方面探索在课堂教学中进行 思辨教学的路径 (王艳, 王一川, 李艺美\&任丽娜) 但是在大学英语课堂教学中还普遍存在 “思辨缺席” 的状况。

\section{4. 大学英语课程中实现思辨教学的路径}

在大学英语教学中有效进行思辨教学应该从以下 几方面入手。

首先, 应厘清思辨教学的属性。在各种教育教学目 标中, 有一部分是显性的, 一部分是隐性的。语言是思 维的载体, 也是认知活动从低级到高级的心理过程的中 介。语言的学习必然有着思维的变化和改变。因此语言 学习和思辨能力是一个相辅相成的过程。语言学习必须 以语言能力的提高为基础, 语言知识的输入和技巧的训 练是显性的教学目标, 应该是基础和前提, 是课程教学 的首要目标。而在这一过程中对思维的锻炼是隐性的, 会伴随着语言学习的全过程。思辨能力的培养应该在学 生语言习得的基础之上, 在习得过程中进行沉浸式教学。 因此思辨能力培养是语言教学的隐性目标。对思辨能力 的培养应顺应语言能力培养, 而不能不顾语言习得规律, 造成语言学习的压力。

其次, 思辨教学的教学设计符合认知规律, 融入到 英语教学环节中。思辨教学应该注重对思辨能力的解构。 根据《德尔菲报告》思辨能力的两个维度分别为认知维 度和情感维度。根据 Bloom 的认知教育目标分为知识、 理解、运用、分析、综合、评价六个认知层次。而这些 目标的认知水平逐层递增。从理解阶段开始就有思辨能 力培养的影子, 对已输入知识的重构 (复述), 解释 (对 知识进行说明和解释) 和推断 (包涵归纳与总结), 这 一阶段是初级的思辨能力培养阶段。在语言课程中, 在 语言知识输入的过程中, 对学生是否理解语言知识, 是 否依赖于语言知识的理解与运用达到理解, 并能够运用 目的语言进行复述和表达自己的意图, 这些都与大学英 语课程教学目标对接。具体的融入课堂的环节如文本阅 读中教师对文本内容进行分析, 对学生进行依赖于文本 内容的提问, 复述和英英释义, 以及依据文本进行的课 后总结 (summary) 等都与这一层面的思辨能力培养相关。 应用阶段涉及更多的思维能力训练, 如抽取有效的知识 单元、分析、重新组织知识片段等。而这一阶段的英语 教学环节在语言输入的基础上, 应该加入从输入的语言 中分解出独立知识模块, 根据相似主题的任务使学生对 输入的语言知识进行简单应用, 达到语言有效输出。教 学环节安排如通过阅读文本, 借用文本结构、观点、语 言等进行与之相关主题的写作或口头表述都体现思辨 能力的培养。分析和综合是比较高级的思维训练, 需要 运用对比、区分、并采用恰当的方法和实施计划来建构 新的知识, 形成判断的基础和体系。这一阶段的英语活 动需要在一定的语言基础之上进行。目前大学英语中提 倡基于任务的学习, 项目学习, 都是锻炼思辨思维的有 
效手段。通过布置与语言学习相关的任务, 学生需要查 找资料、组织资料, 进行观点对比、在众多资料中选取 恰当的材料, 形成观点, 完成任务。这一过程既是思维 发展的过程, 也是通过语言输入、内化和输出的过程即 语言习得的过程。评价阶段是最高的思维发展阶段, 需 要在前几个阶段之上最终形成有理据的价值判断, 比如 辩论、学术论文写作等较为高级的语言输出活动, 都需 要较高的思辨水平才能完成。

思辨能力培养的多层次多阶段特性, 要求大学英语 课程设置要有完善的课程体系, 要分不同层次的课程满 足不同的思维培养和语言习得需求。并针对不同的目标, 明确采取不同的教学手段和模式引导学生在习得语言 能力的同时发展思辨能力。

情感维度则与深度学习相关, 要注重学生的情绪感 受, 教师的问题以及教学方式应该从学生的情感感受出

发, 使学生接受思辨活动的价值、并具有好奇心和探索 心，积极进行思维活动。

最后，教师应该增强自身思辨能力并进行教学反思。 教师对思辨能力培养的认识和自身思辨能力将会制约 课堂思辨教学的有效性。有研究显示教师课堂上提问学 生的方式以及问题认知水平, 对学生的思辨能力促进有 极大的相关性 (杨丽芳, 2015)。有研究者通过案例分 析的方法发现有效的思辨训练需要有具体的目标, 要按 照目标选择教学内容, 又要选择符合目标的教学步骤和 方法 (文秋芳\&孙旻, 2015)。而教师的思维水平, 思辨 能力与元思辨意识本身制约着教师的教学选择和教学 观念。而掌握思辨能构成和框架等相关理论知识和深入 的研究基础, 对于教师选择适合的教学方法及其思辨能 力引导的水平都有至关重要的影响, 因此大学英语教师 的培训以及教师发展计划当中应该重视对思辨能力以 及思辨教学等相关理论和策略的引导。

思辨能力在高等教育中有着重要的意义, 但思辨能 力的培养是一个较为缓慢的过程, 不是一种教学方法和 一门课程能够完成的任务。思辨能力的培养应该在高等 教育的整个体系中并贯穿整个教学过程。而对于大学英 语课程来说, 应该完善课程体系, 丰富课堂教学组织形 式, 明晰目标, 采取有效的教学手段, 在思辨能力培养 中发挥更大的作用。

\section{项目基金}

本文为辽宁省教育厅一般项目《移动环境 下大学生英语学习特征研究及教学模式建构》 (JDW2017002) 的阶段性成果之一。

\section{REFERENCES}

[1] Foundation for critical thinking.2011 Our Concept and feinition of critical thinking.[2011-11-08] http://www.eric.ed.gov
[2] Lin Xiao \& He Lianzhen Critical Thinking cultivation in College English teaching Practice. Journal of Xi'an international studies university. 2017.3 P 61-66

[3] Ma Huarong\&Gong Xiaobin Critical Thinking and Higher Education. Overseas English.2019.11

[4] Jin Xiaohong The Teaching Approach of Cultivating the Critical Thinking Ability in College English Argumentative Composition. Journal of Jiangxi Normal University (Philosophy and Social Sciences Edition. 2018.2

[5] Wang Ting.Research on the "Three-stage Comprehensive Writing Teaching Model" to Cultivate Students' Critical Thinking Ability. Theory and Practice of Education. 2019.6

[6] Zhou yishu An action research on enhancing the teaching effects of college English writing by training of continuation tasks

[7] Foreign Language Education in China.2019.1

[8] Li Yimei\& Ren Lina Research on Deep Reading teaching of college Englih based on the critical thinking development. Shanxi Education. 2019.10

[9] Wen Qiufang\& Sun Min On key issues about critical thinking development in College EFL classroom.

[10] Yang Lifang The cognitive features of reading classroom questioning and critical thinking development. FLC. 2015.3 